\title{
Desafíos para el aula de lengua en el siglo XXI: apuntes para una fundamentación desde una lectura fenomenológica-merlopontiana de la palabra'
}

\author{
Challenges in the XXlst Century Language Class: Notes for a Basis \\ from a Phenomenological and Merleau-Pontian Reading of the Word
}

\author{
VANESA CONDITO \\ Universidad Nacional de Rosario - CONICET \\ Argentina \\ vacondito@hotmail.com
}

(Recibido 3O-O7-2OI4; aceptado II-O3-2OI5)

Resumen.En el presente escrito nos proponemos realizar un trabajo de lectura crítica de la propuesta filosófica fenomenológica de Merleau-Ponty en torno del lenguaje a los fines de problematizar nuestro trabajo docente en el área de Lengua y Literatura y de pensar una posible fundamentación de nuestra praxis. El punto de partida que motiva nuestras indagaciones es el reconocimiento de que atravesamos una coyuntura compleja en que el lenguaje pareciera verse reducido a un código informativo con pretensiones de transparencia y neutralidad-, en detrimento de su valor auténticamente simbólico y expresivo.

Palabras clave: Lenguaje; Postmodernidad; fenomenología; educación; lengua y literatura.
Abstract. In this paper we propose to perform a critical reading assignment of the phenomenological philosophy of MerleauPonty about language in order to problematize our work as teachers in the area of Language \& Literature and to think about a possible foundation our praxis. The starting point motivates our research is the recognition that we are experiencing a complex situation in which language seems to be reduced to an informative code - with claims of transparency and neutrality-, to the detriment of its truly symbolic and expressive value.

Keywords: Language; Postmodernism; Phenomenology; Education; Language \& Literature

\footnotetext{
I Para citar este artículo: Condito, Vanesa (2OI5). Desafíos para el aula de lengua en el siglo XXI: apuntes para una fundamentación desde una lectura fenomenológica-merlopontiana de la palabra. Alabe 11. [www.revistaalabe.com] DOI: IO.I5645/Alabe.20I5.II.8
} 


\title{
I- Introducción, fundamentación y propuesta de trabajo
}

\author{
Por eso cada palabra dice lo que dice \\ y además más y otra cosa
}

Alejandra Pizarnik (1971)

Es sabido que en las últimas décadas asistimos a una profusión de los más variados discursos -docentes, familiares, mediáticos, ministeriales- que se erigen desde el cuestionamiento de las instituciones escolares y, específicamente, proclaman y denuncian las dificultades de los estudiantes (niños y adolescentes) respecto de la lectura, la escritura y la expresión oral. Es decir, si seguimos en detalle este conjunto de voces, opiniones más o menos formadas y reclamos, pareciera que la organización y puesta en palabra por parte de los jóvenes estuviera en crisis y en este contexto la escuela es, si no responsable, al menos ineficaz para colaborar en una posible respuesta.

Ahora bien, no es de nuestro interés aquí establecer una historización del asunto ni detectar si es esta, efectivamente, una problemática reciente o de larga data. Sin embargo, si asumimos que es, en efecto, una crisis emergente de las últimas décadas, cabría que nos interrogáramos: ¿ello qué significa? ¿por qué, precisamente, en un mundo que asegura a todas voces haber conquistado la democratización de la información, la expansión de la polifonía (blogs y redes sociales mediante), la emergencia de una sociedad globalizada del conocimiento (en la que hacer un postgrado - escribir una tesis- y conocer aunque sea rudimentos de una lengua extranjera es casi un mandato para muchos sectores), y que asume la alfabetización como un tema en buena medida saldado, nos encontramos con este multiplicado cuestionamiento de la producción verbal de los estudiantes, tanto de los de la escuela primaria, como de los ingresantes a la universidad...? ¿Constituye esto, realmente, una paradoja? ¿Es un problema que debemos de modo privativo, en nuestro carácter de docentes de lengua, solucionar?

Desde nuestra perspectiva como docentes e investigadores, consideramos que, ciertamente, se evidencia una dificultad cada vez mayor por parte de los jóvenes estudiantes respecto de la asunción de la palabra y de su puesta en funcionamiento, es decir, una dificultad de ejercer una auténtica praxis lingüística (Bronckart, 2007) que les permita hallar, constituir y asumir una voz dentro del concierto heterogéneo de las distintas voces -de las circundantes y de las posibles-. Esto es, por un lado, si partimos de la asunción de que la lengua es esencial e inherentemente permeable a la polifonía (Ducrot, 200I; García Negroni y Tordesillas Colado, 2OOI) y que, por ello, no hay posible enunciado producido que no se constituya como "un eslabón de la cadena, muy complejamente organizada, de otros enunciados” (Bajtín, I982: 255), se desprende que, en principio, el poder hallar y constituir el lugar de enunciación singular y personal lejos está de ser una tarea sencilla. Ahora bien, por otro lado, lo que quisiéramos problematizar aquí es que -más allá de este desafío permanente y difícil labor de constitución de la propia enunciación a que nos 
somete de por sí el lenguaje por su carácter inherentemente polifónico- en el momento presente, en el que la proliferación y expansión de voces anónimas que emergen de los múltiples espacios mediáticos con los que convivimos es parte constitutiva de nuestra realidad cotidiana, se está gestando un contexto aún más complejo y en buena medida poco alentador para la posibilidad de hallar y organizar una voz propia y singular, esto es, una responsabilidad enunciativa que sea consciente y, parafraseando a Ducrot (200I), gestora de 'su decir y lo dicho'.

Por consiguiente, lejos de resultar una paradoja que este problema de la posibilidad de asumir una voz y de apropiarse plenamente de la lengua (desde un conocimiento de sus potencialidades semióticas y retóricas) acontezca en un contexto como el actual, cabría pensar que se trata justamente de su síntoma o emergente. Es decir, en una coyuntura en que estamos permanentemente circundados por mensajes multimedia que se inscriben, la mayoría de las veces, sin autor, sin sujeto o colectivo responsable, anónimos, cual verdades absolutas (como ocultando su carácter de ser construcciones discursivas, queriendo pasar por las cosas mismas); mundo de sintagmas prefabricados que continuamente 'posteamos' o 'compartimos' al infinito en las redes sociales, no analizados ni analizables (cual bloque indescomponible); en un contexto en que pareciera imperar la lógica del 'emoticón’ y la 'etiqueta’ para inscribir estados de ánimo, ideas políticas o, incluso, religiosas y estéticas; momento en que asistimos a la virtualización de las relaciones con el otro, en que asumir la palabra es muchas veces sinónimo de rozar el dedo en una pantalla, nos preguntamos: ¿en qué medida queda margen para que el lenguaje aparezca como constitutivo y constituyente para estos estudiantes que, por una cuestión cronológica, se inscriben desde siempre en estas coordenadas..?, ¿qué vínculo establecen estos sujetos con la palabra, cuando todo tendería a que (lejos de la relación de dominio y proyección que soñó la modernidad) devengan meros usuarios o simples 'operadores’² (en una relación de exterioridad instrumental), y no sujetos de y en el lenguajes?

Si bien en modo alguno sostenemos que esta problemática pueda y deba ser 'solucionada’ de manera exclusiva desde el aula de lengua en las instituciones escolares, sí consideramos que es viable (y necesario) que nos pongamos a pensar en cuál es nuestro rol como docentes, cuyo objeto a enseñar es precisamente la lengua y la literatura, en el marco de esta coyuntura. Una manera interesante y posible de encarar esta reflexión, que aquí quisiéramos esbozar con el objeto de propiciar un ulterior debate, es a partir de la realización de una interrogación de la propuesta fenomenológica de Merleau-Ponty en torno de la palabra. Esto es, a continuación proponemos entablar un diálogo con lo que la filosofía de Merleau-Ponty ${ }^{4}$ tiene para decirnos respecto del lenguaje, de su valor gestual-expresivo, constitutivo y constituyente del mundo que habitamos y que nos convoca,

\footnotetext{
${ }^{2}$ Cfr. Candelero (2012 a.).

${ }^{2}$ Cfr. Benveniste (I986, I987).

${ }^{2}$ Nos centraremos fundamentalmente en la lectura de Fenomenología de la percepción (i975 [i945]), y revisaremos también ciertos aspectos de El mundo de la percepción (2003 [I948]) y Signos (I964).
} 
justamente, a pensar. Lejos de tratarse de una búsqueda de soluciones rápidas capaces de originar una propuesta didáctica secuenciada y de aplicación efectista, lo que aquí intentaremos es indagar de qué manera las reflexiones merlopontianas sobre la palabra pueden operar como fundamento de nuestra labor docente, dada la problemática antes planteada.

Consideramos que un trabajo de esta índole, cuyo atributo central es su carácter ensayístico y, por tanto, orientado a suscitar reflexión y nuevos interrogantes, constituye una labor necesaria al menos por dos motivos. En primer lugar, el fundamento principal reside en las características mismas de la problemática desde la cual iniciamos nuestras indagaciones, esto es, por un lado, el reconocimiento de que a nuestros estudiantes pareciera resultarles cada vez más difícil la posibilidad de reconocer las potencialidades expresivas, significantes y enunciativas del lenguaje y, por otro, la existencia de toda una serie de cuestionamientos y propuestas paliativas orientadas a buscar soluciones que, según nuestro criterio (aunque no dudamos de las posibles buenas intenciones que las generan), no parecen estar analizando los fundamentos de esta crisis. Por consiguiente, un análisis desde otro enfoque del problema, quizás un tanto alejado de la bibliografía y abordaje tradicional con que comúnmente se lo aborda dentro del campo de la lingüística, puede resultar de interés. Así -y este es el segundo motivo que fundamenta la propuesta-, la decisión de problematizar nuestra tarea docente y sus fundamentos en un contexto como el actual a partir de la interrogación de un autor proveniente, en principio, del campo de la filosofía, obedece a la necesidad de abordaje del tema desde un enfoque diverso que, en cuanto tal, nos permita a los docentes que provenimos del ámbito de las ciencias del lenguaje ejercer un principio de indagación filosófica que, en rigor, nos estamos debiendo.

Ahora bien, en particular, la elección de Merleau-Ponty para ejercer este principio de reflexión acerca de los fundamentos e importancia de nuestras prácticas como docentes de lengua se debe, en primer lugar, a que se trata de un autor cuyo pensamiento, como veremos, se organiza a partir de la asunción de que es el lenguaje una de las maneras fundamentales en que el sujeto realiza su existencia. Asimismo, y en segundo lugar, aunque está relativamente reconocido que la fenomenología merlopontiana sobre la palabra constituye un fundamento filosófico de las teorizaciones fundacionales de Benveniste (I986, I987) acerca de la enunciación ${ }^{5}$, quienes nos situamos en el campo de las ciencias del lenguaje -los docentes de lengua y literatura- por lo general no realizamos un trayecto de lectura de tales fundamentos. En consecuencia, aunque Merleau-Ponty no ocupe un lugar privilegiado en la bibliografía de referencia de la mayoría de los docentes del área, resulta fundamental que conozcamos sus principales teorizaciones sobre el lenguaje puesto que, en efecto, son las que operan como basamento de un fructífero campo de estudio como lo es la lingüística de la enunciación.

5 Léase, en esta orientación: Coquet, J-C y Fenoglio, I. (2OI4). “Introducción”. En Benveniste, E. (2OI4). Últimas lecciones. Collège de France 1968-1969. Buenos Aires: Siglo XXI 
A continuación, antes de involucrarnos plenamente en la discusión que proponemos establecer, postularemos, en primer lugar, un breve marco general e introductorio para contextualizar el pensamiento del autor y, específicamente, sus asunciones respecto del lenguaje. A partir de esto último, como dijimos, luego interrogaremos nuestra praxis docente en esta coyuntura postmoderna (Casullo, 1989) -en tanto presente de palabras virtuales, palabras sin cuerpo y palabras de nadie-, con el objeto de comenzar a organizar una reflexión acerca de la problemática y, por qué no, suscitar ulteriores propuestas de trabajo desde estas bases.

\title{
2- Algunos apuntes sobre el pensamiento de Merleau-Ponty
}

\author{
Una mirada desde la alcantarilla \\ puede ser una visión de mundo \\ La rebelión consiste en mirar una rosa \\ hasta pulverizarse los ojos
}

Alejandra Pizarnik (1962)

El pensamiento de Merleau-Ponty acerca del lenguaje se enmarca en una reflexión mucho más abarcativa que involucra a la definición misma de lo humano y, específicamente, a la relación del hombre con el mundo: relación con las cosas y relación con los otros.

Ahora bien, es preciso que tengamos en cuenta que el escenario con el que el autor discute y sobre el cual esgrime sus postulados es el de la tradición filosófica cartesiana. Es decir, la fenomenología merlopontiana supone una manera de concebir al hombre ajena a todo fundamento dualista y racionalista cuyos orígenes, como es sabido, se remontan a Descartes, y son fundacionales de los ideales de la modernidad y de las instituciones por ella fundadas (muchas de las cuales perviven hasta el presente ${ }^{6}$ ). Así, podríamos decir que uno de los cuestionamientos centrales que Merleau-Ponty establece respecto de la tradición cartesiana atañe a la consideración del hombre en términos de una dualidad mente (res cogitans) / cuerpo (res extensa), bajo el predominio lógico de la conciencia o razón, en tanto que es desde allí que se organiza el mismo mundo: como proyección de lo que esa conciencia puede instaurar, clasificar y programar en relación con las cosas, siempre inertes y plegadas sobre sí.

Frente a ello, en primer lugar, Merleau-Ponty señala categóricamente que "el mundo está previamente a cualquier análisis que yo pueda hacer del mismo” (1975: 9). Es decir, no se trata de una proyección de lo que uno es, sino que precede y, desde allí nos afecta y convoca a ser, en tanto que lo habitamos:

\footnotetext{
${ }^{6}$ Pensemos en la ciencia como institución, o en el mismo sistema educativo (que aunque han presentado importantes cambios, hay todavía cierto sustrato moderno en ambas instituciones).
} 
El mundo no es un objeto cuya ley de constitución yo tendría en mi poder; es el medio natural y el campo de todos mis pensamientos y de todas mis percepciones explícitas. La verdad no "habita" únicamente el "hombre interior"; mejor aún, no hay hombre interior, el hombre está en el mundo, es en el mundo que se conoce (Merleau-Ponty, I975: Io)

Esto es así puesto que para el autor se parte de una noción totalmente distinta tanto del hombre como de las cosas del mundo; es decir, dado que se los piensa de otra manera, el tipo de vínculo que se establecerá entre ambos es, en efecto, de otra naturaleza a la que se planteaba desde el racionalismo cartesiano.

En primer lugar, Merleau-Ponty corre el eje puesto en la razón y, frente a ello sostiene el predominio de la percepción en la definición de lo humano: percepción en tanto que actividad consciente en que se ve involucrada, no ya 'la mente' o 'el pensamiento puro', sino el cuerpo en su totalidad, el cuerpo visto como unidad compleja o cuerpo consiente:

Si hablamos de 'unidad' y no de identidad es porque se reconoce diferenciación, pero ya no de partes -que siempre son sólo unidades más pequeñas- sino de aspectos, o mejor, de fases o momentos. Conciencia y cuerpo son complementarios: no hay conciencia sin cuerpo que la habilite, ni cuerpo sin conciencia que le aventure [destacados en el original] (Candelero, 2OIr: O2)

En otras palabras, en este planteo estamos ante un hombre que no tiene un cuerpo sino que es un cuerpo ${ }^{7}$, y siempre un cuerpo actuante, intencional, expresivo y cognoscente. Esto es así puesto que el hombre puede sino ser/estar en el mundo con el que interactúa permanentemente -el cuerpo es mediador del mundo ${ }^{8}$ - y a partir de lo cual organiza el conocimiento: conocimiento pre conceptual y sinestésico, que para MerleauPonty es previo al saber conceptual-científico, que aquí no es negado sino puesto en un segundo plano (dado que se genera onto y filogenéticamente de manera posterior).

En segundo lugar, como dijimos, en Merleau-Ponty no sólo hay un modo diametralmente opuesto de concebir a lo humano, sino también a las mismas 'cosas' o 'entes' del mundo, con las cuales el hombre establece el tipo de relación mencionado. Así, si tuviéramos que sintetizar la manera en que se inscribe la consideración de las cosas, podríamos decir que se las plantea en términos de gestos, es decir, lejos de ser materia inerte o res extensa, el mundo está constituido por otros-cuerpos o acontecimientos sensibles que manifiestan siempre una apertura y una expresividad para el sujeto que percibe: "fenomenológicamente observado, el otro no es prioritariamente extensión, cosa extensa, sino

\footnotetext{
${ }^{7}$ Cfr.: "yo no estoy delante de mi cuerpo, estoy en mi cuerpo, o mejor, soy mi cuerpo" (Merleau-Ponty, I975: I67)

${ }^{8}$ Cfr: "El cuerpo es nuestro medio general de poseer un mundo" (Merleau-Ponty, I975:I62)
} 
comportamiento, gesto. Todo cuerpo es dinamismo: en modo alguno cosa ahí, plegada sobre sí y dispuesta, sino acontecimiento sensible que se sale de sí y actúa” (Candelero, 2OII: O7).

Y, justamente, en función de esa expresividad y gestualidad de las cosas-cuerpo, podemos decir que no pueden sino estar dotadas de sentido:

Las cosas no son simples "objetos neutros" que contemplamos; cada una de ellas simboliza para nosotros cierta conducta que nos evoca, provoca por nuestra parte conductas favorables o desfavorables (...) Nuestra relación con las cosas no es una relación distante, cada una de ellas habla a nuestro cuerpo y nuestra vida (...). El hombre está envuelto en las cosas y éstas están investidas en él (Merleau-Ponty, 2OO3: 3O-3I)

Ahora bien, para comprender mejor su planteo, es fundamental aclarar que, para este punto de vista, el sentido - de los gestos del mundo y del propio cuerpo, en tanto ambos están dotados de gestualidad expresiva- es inseparable de la manera en que se muestra, puesto que (y esto tendrá, como luego veremos, consecuencias respecto del modo de entender el lenguaje) forma y contenido, figura y fondo, tema y estilo son indisociables: "En este mundo es imposible separar las cosas y su modo de manifestarse" (MerleauPonty, 2003: 6o).

Podemos sintetizar lo expuesto diciendo que desde aquí se postula una centralidad del cuerpo en el proceso de conocimiento, pero del cuerpo entendido como subjetividad encarnada, gestual, expresiva e intencional que percibe activamente el mundo (como parte de él), en tanto espacio reticular de gestualidades y significaciones, siempre dinámico y abierto. En palabras del autor:

Por estar en el mundo estamos condenados al sentido; y no podemos hacer nada, no podemos decir nada que no tome un nombre en la historia (...). El mundo fenomenológico es, no ser puro, sino el sentido que se transparenta en la intersección de mis experiencias y en la intersección con mis experiencias con las del otro (MerleauPonty, r975: 19)

Ahora bien, es preciso que consideremos que la reflexión sobre el lenguaje propuesta por Merleau-Ponty tiene lugar en el contexto de esta comprensión del cuerpo como expresión y gestualidad significante. Es decir, su reflexión en torno del lenguaje -que aquí quisiéramos retomar y repensar en el marco de nuestra praxis docente en el presente siglo XXI (cuya problemática fue esbozada en la introducción) - se inscribe como parte integrante de su concepción de hombre y de mundo. Como veremos en el siguiente subapartado, en el análisis filosófico de Merleau-Ponty sobre la palabra, ésta es concebida, principalmente, como una de las maneras en que un sujeto encarnado realiza su existencia, simultáneamente sensible y racional, simultáneamente biológica y cultural, entre lo individual y lo colectivo, entre lo dado y lo por darse. 


\title{
3 - Una interrogación acerca del lenguaje desde la lectura de Merleau-Ponty
}

\author{
sólo palabras \\ las de la infancia \\ las de la muerte \\ las de la noche de los cuerpos
}

Alejandra Pizarnik (1971)

Para comenzar, resulta fundamental que partamos de una asunción de base sobre la que se erige toda indagación y caracterización del lenguaje por parte de MerleauPonty: el lenguaje es, aquí, también considerado en términos de cuerpo. Es decir, como puede verse, la fenomenología merlopontiana asume un cuestionamiento radical del dualismo cartesiano, y esto tendrá sus consecuencias también en la manera en que se piense la palabra.

Ello implica, en primer lugar, que para el autor el lenguaje no tiene un más allá que le otorgue fundamento y que opere como origen: así como no es posible concebir una conciencia sin cuerpo, el lenguaje -en tanto también es cuerpo- no es un mero representante del pensamiento, entendido éste como una esencia primaria y fuente de lo dicho, porque, en efecto, no hay pensamiento posible sin lenguaje que lo encarne. Dice, categóricamente Merleau-Ponty: "el discurso no traduce, en el que habla, un pensamiento ya hecho, sino que lo consuma” (I975: 195). En otras palabras, es en, pory desde el lenguaje que podemos pensar y, sobre todo, ser conscientes de lo que pensamos, todo ello como parte de un mismo movimiento y como parte consustancial de lo que somos en tanto no podemos sino constituirnos y ser plenamente a partir de las palabras de nuestra lengua materna, que arman nuestro mundo y lo habitan como integrantes de nuestro cuerpo ${ }^{9}$ :

Si la tematización de lo significado no precede a la palabra es porque es su resultado (...) Expresar, para el sujeto hablante, es tomar conciencia. No expresa solamente para los demás, expresa para saber él mismo lo que se propone (Merleau-Ponty, I964: I08).

Así pues, se evidencia que para este punto de vista la palabra, en tanto cuerpo, no recibe desde afuera su sentido (como si fuera su vestimenta o disfraz): hay un sentido gestual inmanente de la palabra que, desde su misma materialidad significante y entonación estilística, materializa un sentido y se inscribe como ser de y en el mundo que siempre

\footnotetext{
9 Hay una escena en Padre Padrone (r977), película de los hermanos Taviani, que ilustra lo que pretendemos formular: cuando el pequeño Gavino, protagonista de sólo seis años destinado a vivir en la soledad de los montes de Cerdeña cuidando cabras, intenta con muchísima dificultad cargar un gran balde de leche, su padre le dice "- anda, fuerza, ayúdate con la voz", instándolo a que cargara el pesado balde acompañado de gritos de valor dirigidos hacia sí mismo, como parte de un mismo movimiento: porque el cuerpo con brazos, piernas y voz que debe levantar el balde es, indudablemente, el mismo y único (y, de hecho, finalmente, palabra-grito mediante, puede levantar el balde).
} 
tiende hacia otro y, por ello, permite entablar una comunicación o diálogo. En términos de Merleau-Ponty: "La palabra es un verdadero gesto y contiene su sentido como el gesto contiene el suyo. Es esto lo que posibilita la comunicación” (I975: 2OI).

Ahora bien, es necesario aclarar que el hecho de que la palabra posibilite la comunicación intersubjetiva no quiere decir que sea ésta su razón de ser y único valor, ni mucho menos que opere como un mero medio de ‘transmisión de información': "Mucho más que un medio, el lenguaje es algo así como un ser" (Merleau-Ponty, ı64: 53). Esto es, en tanto que ser gestual, la palabra permite entablar un diálogo con otros, pero esto es así porque primeramente es la que nos posibilita, como vimos más arriba, pensar y tener conciencia de lo pensado, tener conciencia del mundo que habitamos y que solo existe realmente para nosotros desde que lo podemos nombrar: "la denominación de los objetos no viene luego del reconocimiento, es el mismo reconocimiento (...) Nombrar el objeto es hacerlo existir o modificarlo" (Merleau-Ponty, I975: 195).

Este último aspecto, podríamos decir, performativo y fundacional del lenguaje ${ }^{\mathrm{IO}}$, se desprende también como consecuencia de su ser inherentemente corpóreo, y de su carácter organizador del pensamiento. Es decir, la palabra es el cuerpo del pensamiento en el sentido de que nos permite tener conciencia de los hechos, de las cosas, de la multiplicidad de lo percibido en el mundo en que existimos, a partir de la posibilidad que tenemos de nombrarlo y de predicar sobre ello: "no hay modo de saber de un hecho de no ser por la mediación de esas obras que son los signos" (Candelero, 20I2 b: 69). En otras palabras, sólo en y por el lenguaje nos apropiamos de la heterogeneidad de lo vivido y le otorgamos un sentido, lo cual es fundamental para que las cosas existan (recordemos que para la perspectiva fenomenológica no hay percepción sino para alguien: punto de vista del sujeto que percibe).

Asimismo, quisiéramos precisar también que, desde aquí, se entiende a este otorgar sentido como un dar forma y valor a las cosas, hechos, estímulos y situaciones que nos rodean y con las cuales interactuamos permanentemente. Ahora bien, obsérvese que en toda actividad de formalización es necesario que exista, como mínimo, una previa operación (consciente o no) de selección. Es decir, no es posible nunca decirlo todo y desde todos los puntos de vista posibles e imaginables: la realidad es heterogénea, dinámica y compleja, sobre la cual el lenguaje -sometido a la linealidad del significante (Saussure, І9І6) - efectúa un necesario recorte, selección y organización en virtud de un punto de vista. Justamente por ello es que podemos afirmar que el lenguaje es siempre sinecdóquico (Pérez-Rogieri, 2012), y nuestra percepción de las cosas sobre las que a partir de éste podemos pensar y actuar, también lo es: “saber es siempre saber una parte” (Candelero, 2OI2 b: 7I).

En este sentido, cada lengua en particular, en tanto manifestación concreta, situada e histórica de la capacidad humana de simbolizar, implica ya una primera sinécdoque del mundo: una cosmovisión particular que, para los hablantes nativos de dicha lengua,

io $C f$ r. Todorov, T. (1972). 
suele ser naturalizada (aunque en modo alguno lo sea), y que opera como base para las posteriores reacentuaciones ${ }^{\mathrm{II}}$ y sucesivos nuevos y específicos recortes que en las heterogéneas interacciones dialógicas efectivamente acontecen ${ }^{12}$.

Por consiguiente, desde esta perspectiva fenomenológica merlopontiana de la palabra, se presenta una fuerte crítica de la idea del lenguaje como medio transparente que refleja y traduce lo dado, en pos de asumir su entidad significante plena, autónoma, opaca, expresiva y oblicua. El lenguaje es aquí mostración particular de las cosas y del pensamiento, antes que un mero medio de reproducción de lo preexistente:

La palabra no es un medio al servicio de un exterior, tiene en sí misma su regla de empleo, su moral, su visión de mundo, de la misma manera en que un gesto contiene a veces toda la verdad del hombre (Merleau-Ponty, 2003: 9I).

Pues bien, luego de este recorrido, quisiéramos ahora detenernos en la importancia que supone que como docentes de lengua seamos conscientes de este valor de la palabra que nos recuerda Merleau-Ponty, sobre todo en un contexto en el que pareciera, en muchísimos ámbitos, primar un reduccionismo en torno del lenguaje que lo limita a su valor de código representante y transparente de lo que hay y de lo que es, es decir, un valor puramente funcional, instrumental e informativo.

Así, cuando leemos los titulares de un diario -en papel o digital-, o miramos un noticiero (con sus 'zócalos' en mayúscula reiterándose ad infinitum), cuando la supuesta 'heroína' de la teleserie del prime time repite cual marioneta palabras vacías o clichés que simulan expresividad, o cuando una red social nos hace elegir un 'estado sentimental' dentro de una serie de opciones predeterminadas ('soltero', 'comprometido', 'en una relación abierta', ‘casado’, etc.), por sólo mencionar algunos ejemplos, pareciera que el único rol que el lenguaje tuviera encomendado es el de reproducir -de la manera más 'neutra' e impersonal posible, porque así se asegura la transparencia de la reproducciónlo que previamente ya existe (o existió) de una vez y para siempre, de modo homogéneo e idéntico a sí mismo. Y de este modo, lo que no estaríamos considerando es, justamente, el valor corpóreo, gestual, performativo y plurisignificante de la palabra que encarna lo que dice y le da un valor, siempre singular, siempre con una entonación y estilo particular. Tampoco estamos, en estos casos, considerando o asumiendo el valor que la palabra, también en tanto que cuerpo, nos posibilita como sujetos con deseos, intenciones, juicios de valor y criterios singulares, desde el momento es que en función de su gestualidad inherente -es en ella y a partir de ella- que organizamos nuestra conciencia (que es siempre conciencia-de-algo, conciencia-de-mundo).

\footnotetext{
II Léase el concepto de ‘reacentuación’ tonal y valorativa en Voloshinov, V. (I999). Freudismo. Un bosquejo crítico [1927]. Buenos Aires: Paidós.

${ }^{12}$ Volveremos más adelante sobre esta tensión entre lo preestablecido y lo nuevo en el lenguaje.
} 
En este contexto, necesario es que como docentes de lengua organicemos nuestra labor en virtud de que los estudiantes (cercados, en cierta forma, por estas apócrifas representantes de la palabra) se permitan, por un lado, tener conciencia lingüística, lo cual sólo es posible, en buena medida, tomando cierta distancia, desarmando, desandando, analizando y, por qué no, jugando con la lengua materna para luego reapropiárnosla en todo su espesor.

Si nuestro fundamento y horizonte de trabajo se orienta por este necesario objetivo de la 'toma de conciencia lingüística', se desprende que no hay motivo alguno para erradicar de la clase de lengua el trabajo explícito de análisis fonológico, morfológico y sintáctico, como aconteció en la década del '9o, sin demasiado fundamento, en el currículum de la Escuela Media en Argentina -y frente a lo cual actualmente no pareciera haber una propuesta coherente para contrarrestar dicho vacío ${ }^{\mathrm{I} 3}-$. Esto es, en un contexto de palabras 'a seleccionar' y de enunciados prefabricados, ejercer un trabajo analítico resulta una tarea prioritaria para nuestras clases, e incluso una propuesta, en cierta medida, 'revolucionaria'. Nos referimos a un trabajo analítico, eso sí, que se oriente desde un movimiento dialéctico entre la forma y el sentido (siempre situado, siempre ideológico), entre la norma y la reacentuación, entre la deconstrucción y la construcción, entre la estandarización y el juego de palabras, entre la lectura silenciosa hermenéutica y la lectura en voz alta que resalte el valor gestual del plano fónico de la lengua; es decir, una propuesta de análisis que devuelva el valor simultáneamente semiótico-formal y argumentativoexpresivo de la palabra, que se halla en las antípodas de las propuestas de cuño escolástico ${ }^{\mathrm{I} 4}$ con que se trabajó durante décadas y que motivó la erradicación de la gramática de nuestras aulas -prácticas rutinarias, orientadas unidireccionalmente de la norma al caso, y centradas sólo en la forma pura de la lengua-.

Por otro lado, también es menester que, frente a esta problemática, permitamos, desde nuestro lugar como profesores, generar instancias en que los jóvenes se permitan asumir su lugar de ser hablantes-pensantes, es decir, de ejercer la conciencia de su ser seres de palabra, sujetos capaces de pensar y actuar a partir del lenguaje. Es decir, cabría que nos preguntáramos, ¿por qué, en lugar de plantear trabajos de escritura y debate en primera persona nos la pasamos entregando cuestionarios o guías de comprobación de lo leído cuyo valor reside antes que nada en insistir en una idea de lenguaje como reproductor de lo dado? Sin negar el valor que, eventualmente, pueda llegar a tener un trabajo de sistematización de algún contenido conceptual a partir de una 'guía de lectura', lo que estamos queriendo enfatizar es que la especificidad de nuestro trabajo como profesores de lengua debería estar orientado a esa posibilidad de ofrecerle a los estudiantes instancias de trabajo en que se vean motivados y, por qué no, desafiados a asumir una voz propia y enunciar-organizar su pensamiento en primera persona -en permanente y necesaria con-

\footnotetext{
${ }^{\mathrm{I}} 3$ Cfr. Condito, V. (2OI4).

${ }^{\text {I4 }}$ Cfr. Bronckart, J-P. (2007)
} 
frontación con las voces que los rodean (para lo cual, en efecto, un aula constituida por entre veinte y treinta individualidades, es ya un espacio de por sí propicio para advertir la tensión entre polifonía y asunción de la singularidad en y por el lenguaje)-.

Así, si consideramos los planteos de Merleau-Ponty en torno del lenguaje, no podemos no hacernos cargo de que si les enseñamos a nuestros alumnos a palpar y reconocer las múltiples aristas que tiene la lengua, les estamos intentando enseñar, nada más y nada menos, que a pensar, a ejercer y enunciar su palabra-pensamiento y a reconocer que la verdad no está 'allí afuera’ plegada sobre sí y a la espera de que la recojamos mediante palabras, sino que la construimos discursiva, afectiva e intencionalmente, más allá de que por todos lados (sobre todo por la multimedia, nunca ingenua) se intente propiciar lo contrario.

Finalmente, quisiéramos a continuación precisar brevemente otro aspecto fundamental dentro el pensamiento de Merleau-Ponty en torno del lenguaje, dado que consideramos que se trata de un punto central para organizar las reflexiones que aquí nos proponemos establecer como fundamento de nuestra labor docente: la tensión entre la palabra representativa y la palabra expresiva, entre el ser hablados y ser hablantes, entre lo cultural que nos precede y la posibilidad de reacentuación significante, entre lo dado y lo por darse.

Así, obsérvese que para enfatizar la idea de que el lenguaje es mostración pura y no mecanismo secundario, sustituto de un supuesto pensamiento primario, MerleauPonty retoma como argumento, en principio, las asunciones fundacionales de Ferdinand de Saussure (I9ı6) en el campo de la lingüística (teniendo en cuenta todas las proyecciones que en las ciencias humanas ha tenido a mediados del siglo $X^{15}$ ), específicamente en lo que concierne al valor negativo, inmanente y diferencial de los signos: para Saussure su identidad se da dentro del sistema lingüístico porque un signo es lo que los otros no son. Sin embargo, aunque Merleau-Ponty parta de esta asunción saussureana del valor inmanente, arbitrario y diferencial de los signos lingüísticos (que, ciertamente, le sirve al autor para establecer un cuestionamiento de la idea de lenguaje como representante secundario del pensamiento, o del lenguaje como copia transparente de lo que el mundo exterior 'es'), su lectura fenomenológica trasciende dicha asunción, esto es, va más allá de ella. Es decir, cuando hablamos, dice el autor, pareciera que lo que prima en la palabra no es tanto (o al menos no sólo) su valor diferencial (por referencia opositiva a otras palabras) e inmanente al sistema (que le otorga un valor relativamente estable y siempre idéntico a sí mismo), sino que, antes bien, "la significación devora los signos" (MerleauPonty, r964:95), por lo que cada acto de expresión busca trascender los límites de lo dado o instituido por ese sistema lingüístico -cerrado y autoequivalente- que nos antecede: "Basta que nos prestemos a su vida, a su movimiento de diferenciación y articulación, a su elocuente gesticulación. Hay, pues, una elocuente opacidad del lenguaje” (MerleauPonty, I964:52).

${ }^{\mathrm{I}}$ Cfr. Milner, J-C. (2003). 
Justamente, lo que Merleau-Ponty intenta dejar planteado, y que aquí quisiéramos enfatizar, es que en toda producción discursiva, en toda palabra puesta a funcionar (escrita, oral o en el silencio del pensamiento) siempre hay, en mayor o en menor medida -dependiendo del contexto y del ámbito discursivo-, una dialéctica entre lo dado, lo recibido culturalmente, lo ya dicho, y lo singular, lo creativo, lo nuevo, lo por decir. Es decir, pese a que las lenguas (como toda gestualidad, en general) no son naturales sino distintos modos culturales en que organizamos el mundo ${ }^{16}-\mathrm{y}$ que, en cuanto tales, siempre nos preexisten, nos hablan y hacen hablar desde lo dado-, es importante tener en cuenta que además de este valor inmanente al sistema lingüístico, que podríamos denominar valor conceptual de la palabra, existe también lo que Merleau-Ponty denomina su valor y sentido gestual o expresivo: "Su representación conceptual se deriva, es secundaria respecto de su significación gestual” (I975: I36). Esto es, filo y ontogenéticamente el valor expresivo es, como adelantamos, primario.

En efecto, es este valor expresivo el que particularmente nos evoca -en tanto consumación- la literatura, en la que, como en cualquier obra de arte, se patentiza de modo mucho más explícito que en otras obras-cosas que los signos encarnan el sentido y éste siempre oscila entre lo conocido y lo por conocer. Dicho de otro modo, aunque nunca pueda del todo dejar de partir desde lo instituido (lo compartido culturalmente, lo que forma parte del sistema de la lengua), la literatura advierte de modo sutil que el lenguaje siempre puede salirse un poco más allá de lo establecido, siempre puede devenir lenguaje conquistadory productivo, y no sólo reproductivo.

No obstante, como sostiene Candelero (2OI2 b.) en relación con esta dualidad en tensión constitutiva de la palabra, cabría observar que en numerosas oportunidades, sobre todo en el contexto presente que venimos describiendo y sobre el cual intentamos problematizar nuestro rol docente: "Los hombres solemos escondernos detrás de las palabras, más que darnos a conocer; esa obra -la representativa-, habla por nosotros más que de nosotros" (Candelero, 20I2 b: 42).

Es decir, aunque desde la lectura fenomenológica toda palabra, en tanto cosa obra$d a$, implique una dialéctica entre lo conocido y lo nuevo, y aunque todo sujeto constituido por el lenguaje sea, simultáneamente, un ser hablado y un ser hablante, no siempre funcionamos de este modo puesto que en ocasiones pareciera que el valor expresivo de la palabra permanece meramente latente y no lo asumimos o no lo podemos asumir como tal.

Justamente, consideramos que aquí es donde nuestra labor docente también debería jugar un papel importante e intentar trabajar y sostener su voluntad de cambio y transformación. Ante el imperio de una Wikipedia que es lengua de nadie e ilusión de referencia y representación pura, ante la sobreabundancia de manuales escolares que no son sino repetición velada de lo alguna vez dicho, que sólo parecieran propiciar la lógica del 'corte y pegue' y de palabras despojadas de su impulso expresivo originario -orga-

I6 Por ello es que, según el autor, nunca se puede realmente poseer dos lenguas: "para asumir una lengua [extranjera] por completo habría que asumir el mundo que ella expresa, y nadie pertenece a dos mundos a la vez" (MerleauPonty, 1975: 204) 
nizando textos que se nos aparecen casi como el monstruo creado por el Dr. Frankenstein $^{17}$-; en este contexto, aunque pueda parecer hiperbólico, creemos que resulta de urgencia que mostremos que esa no es la única manera de ser de el lenguaje sino que, de hecho, es solo una y, por cierto, bastante pobre.

Aquí es donde, en efecto, la literatura debe hacerse presente en el aula en tanto lenguaje siempre conquistador (y no, conquistado) capaz de enseñarnos por su sola presencia este valor expresivo, performativo y creador del lenguaje: y decimos presencia de la literatura y no (como lamentablemente suele ocurrir en muchas de nuestras clases) comentarios sobre la literatura. En este sentido, se puede proyectar a la palabra y a la literatura en particular lo que afirma categóricamente Merleau-Ponty en relación con las cosas (en general): "Ninguna definición, ningún análisis, por preciso que retroactivamente pueda ser y para hacer el inventario de tal experiencia, podría reemplazar la experiencia perceptiva y directa que hago de ella" (2003: 6I). Por consiguiente, aunque no negamos el valor que pueda llegar a tener (ni los imperativos curriculares que muchas veces nos lo demandan y ante los cuales no siempre tenemos demasiada libertad) el problematizar conceptualmente aspectos estéticos, históricos o culturales de determinado texto literario, lo que queremos enfatizar es que ello no debería operar en desmedro de la efectiva lectura y vivencia de la palabra literaria en todo su espesor y que, en cuanto tal, silenciosamente (y en ocasiones no tan silenciosamente) se enfrenta a los discursos hegemónicos.

Esto es así dado que, desde esta perspectiva con la que intentamos dialogar, para conocer el cuerpo (el propio, pero también el cuerpo de las cosas en toda su abierta gestualidad) no queda otra que vivirlo ${ }^{18}$, y las palabras son, como vimos, fundamentalmente cuerpos que es preciso que habitemos si no queremos ser ocultados tras esas 'apócrifas representantes de la palabra' a las que aludíamos anteriormente y que insisten en dominar a fuerza de su reiteración y multiplicación.

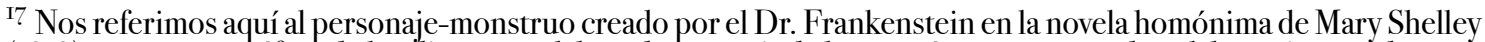
(I8I8), en tanto metáfora de los discursos elaborados a partir de heterogéneos recortes de palabras ajenas y descontextualizadas con los que comúnmente nos encontramos en nuestras aulas (y fuera de ellas).

18 “ya se trate del cuerpo del otro, o del mío propio, no dispongo de ningún otro medio de conocer el cuerpo humano más que el de vivirlo" (Merleau-Ponty, I975:215).
} 


\title{
4-A modo de conclusión: palabras finales y hacia nuevos desafíos
}

\author{
Y cuando es de noche, siempre, \\ una tribu de palabras mutiladas \\ busca asilo en mi garganta, \\ para que no canten ellos, \\ los funestos, los dueños del silencio.
}

Alejandra Pizarnik (1965)

En este espacio hemos permitido interrogarnos acerca de ciertos problemas en torno del lenguaje que nos suscita nuestra coyuntura y, en particular, nuestro trabajo docente, a partir de un diálogo con la propuesta fenomenológica de Merleau-Ponty. Como dijimos en la introducción, no se trata de buscar una nueva 'receta mágica y efectista' para solucionar de una vez y para siempre las dificultades que encontramos en nuestra labor (y fuera de ella). Por lo contrario, el eje consistió, a partir de un trabajo de indagación y lectura crítica con carácter ensayístico respecto de las formulaciones merlopontianas en torno del lenguaje, en intentar problematizar el fundamento de nuestro accionar en el marco de un contexto complejo que, según nuestro criterio, no hace más que opacar y eclipsar el valor corpóreo, gestual y expresivo del lenguaje y la posibilidad de que los jóvenes se constituyan plenamente como hablantes o enunciadores conscientes.

En consecuencia, consideramos que las reflexiones delineadas en el presente estudio -las que, como adelantamos, suponen un punto de partida para repensar posibles estrategias didácticas a partir de un sustento filosófico- nos permiten comenzar a preguntarnos si desde nuestro lugar como profesores de lengua estamos o no propiciando un trabajo de reflexión en torno del lenguaje que aunque sea discuta o ponga mínimamente en entredicho el valor que desde múltiples sectores se está (creemos que no de modo ingenuo) favoreciendo, y que estaría en las antípodas de lo que Merleau-Ponty nos dice al respecto.

Desde aquí, creemos que el aporte de las reflexiones de Merleau-Ponty reside en que, justamente, nos recuerda que es posible, a partir de un trabajo de distanciamiento, reconocimiento, deconstrucción lógica y juego poético con el lenguaje, aun en esta coyuntura, volver a ser (cuerpos) conscientes de la palabra y encontrar nuestra voz-siempre en tensión entre lo dicho y lo por decir, entre la polifonía constitutiva del lenguaje y la posibilidad de reacentuar la singularidad- dentro del concierto de voces anónimas que nos rodean (acaso las de 'los funestos, dueños del silencio', como dijera Pizarnik): “Lo que queremos decir no está ante nosotros, fuera de toda palabra, como un puro significado. No es más que el exceso de lo que nosotros vivimos sobre lo que ya ha sido dicho" (MerleauPonty, I964: 97).

En ello reside, en buena medida, nuestro desafío. Esperamos que desde este conjunto de presupuestos hayamos podido, aunque sea, comenzar a movilizar nuevos interrogantes y posibles líneas de acción que, evidentemente, necesitamos poner en movimiento. 


\section{Referencias Bibliográficas}

- Bajtín, M. (I982). Estética de la creación verbal. México: Siglo XXI.

- Benveniste, E. (1986). Problemas de lingüistica general I. México: Siglo XXI.

• Benveniste, E. (1987). Problemas de lingüística general II. México: Siglo XXI.

- Bronckart, J-P. (2007) Desarrollo del lenguaje y didáctica de las lenguas. Buenos aires: Miño y Dávila.

- Candelero, N. (2OII). Merleau-Ponty, distinto a Descartes. Material de Cátedra correspondiente a parte de la Tesis Doctoral de Candelero, N: Experiencia científica / Experiencia religiosa. Posibilidades e imposibilidades de una descripción filosófica. Facultad de Humanidades y Artes (UNR), 2012.

- Candelero, N. (2OI2 a.). Postmodernidad. El gobierno de las máquinas / Los cartoons. En Ciencia, Arte y Religión: Observaciones filosóficas 2. Rosario: Ciudad Gótica, I2-25.

- Candelero, N. (20I2 b.). Seis observaciones sobre la palabra. En Ciencia, Arte y Religión: Observaciones filosóficas 2. Rosario: Ciudad Gótica, 37-Ioo.

- Casullo, N. (comp.) (1989). El debate modernidad-postmodernidad. Buenos Aires: Puntosur.

- Condito, V. (2014). “La gramática como problema. Un análisis de materiales didácticos propuestos para el trabajo en el aula de lengua en la escuela media”. Ponencia presentada en el Congreso Nacional Cátedra Unesco para el mejoramiento de la calidad y equidad de la educación en América Latina, con base en Lectura y Escritura, I6 y I7 de octubre de 20I4, Rosario (Argentina). Publicación en Actas en prensa.

- Coquet, J-C y Fenoglio, I. (2OI4). “Introducción”. En Benveniste, E. (2OI4). Últimas lecciones. Collège de France r968-1969. Buenos Aires: Siglo XXI.

- Dasilva, F. (20IO). El pensamiento de Merleau-Ponty: la importancia de la percepción. En Miriada, 6, 93-ı8. Obtenido el 30 de junio desde http://p3.usal.edu.ar/index.php/miriada/article/view/25

- Ducrot, O. (200I). Eldeciry lo dicho. Buenos Aires: Edicial.

- Escribano, X. (2000). Gesto y palabra: la esencia emocional del lenguaje según Maurice Merleau-Ponty. En Emociones Thémata, 25, I75-185. Obtenido el 25 de junio desde http:// institucional.us.es/revistas/themata/25/18\%20escribano.pdf 
- García Negroni, M. M. y Tordesillas Colado, M. (200I). La enunciación en la lengua. De la deixis a la polifonía. Madrid: Gredos.

- Milner, J-C. (2003). El periplo estructural. Buenos Aires: Amorrortu.

- Pérez, L. y Rogieri, P. (Dirs.) (20I2). Retóricas del decir. Lenguaje, verdad y creencia en la escritura académica. Rosario: FHUMYAR Ediciones.

- Saussure, F. (200I). Curso de Lingü̈́stica general [ı9ı6] Buenos Aires: Losada.

- Todorov, T. (г972). "Introducción” en AA. VV (i972). Lo verosímil. Buenos aires: Ed. Tiempo Contemporáneo.

- Voloshinov, V. (1999). Freudismo. Un bosquejo crítico [1927]. Buenos Aires: Paidós.

\section{Fuentes}

- Merleau-Ponty, M. (ig64). Signos. Barcelona: Seix Barral.

- Merleau-Ponty, M. (i975). Fenomenología de la percepción [1945]. Barcelona: Península Ed.

- Merleau-Ponty, M. (2003). El mundo de la percepción. Siete conferencias [1948]. Buenos Aires: Fondo de Cultura Económica. 